\title{
Adding configuration interaction to the time-dependent Hartree grid approximation
}

\author{
José Campos-Martínez and Rob D. Coalson ${ }^{\text {a) }}$ \\ Department of Chemistry, University of Pittsburgh, Pittsburgh, Pennsylvania 15260
}

(Received 24 April 1990; accepted 13 June 1990)

\begin{abstract}
The time-dependent Hartree grid (TDHG) method is extended into an $a b$ initio algorithm for obtaining exact quantum wave packet dynamics. The new algorithm employs a superposition of orthogonal zeroth order time-dependent basis functions generated from a single TDHG wave packet trajectory. The superposition coefficients are themselves time-dependent, and are responsible for mixing the basis functions in such a way as to represent exact solutions of the time-dependent Schrodinger equation. Evolution of the superposition coefficients is governed by a set of first-order linearly coupled ordinary differential equations. The couplings between coefficients are given by matrix elements of a naturally identified interaction potential taken between members of the zeroth order basis. In numerical tests involving computation of $S$ matrix elements for collinear inelastic atom-Morse oscillator scattering the method proves accurate, flexible and efficient, and appears to be easily extendable to more complicated systems.
\end{abstract}

\section{INTRODUCTION}

It is curious that, historically, most computational techniques for extracting detailed quantum mechanical transition rates associated with molecular collisions, light scattering, etc. have focused on solving the time independent Schrodinger equation. ${ }^{1,2}$ Information about the temporal evolution of an initially prepared system can then be extracted from the appropriate set of Schrodinger eigenfunctions and eigenvalues via standard recipes. Although in many cases it is intuitively useful to explicitly follow the spatiotemporal evolution of the initial wave packet state, technical difficulties associated with direct solution of the time-dependent many-body Schrodinger equation were long considered prohibitive. In the last fifteen years the outlook for directly propagating time-dependent wave packets has brightened considerably. A seminal step in this direction was provided by Heller and coworkers, ${ }^{3}$ who developed techniques for propagating multidimensional Gaussian wave packets, showed how to extract state-to-state quantum mechanical transition probabilities ( $S$-matrix elements for scattering processes, photodissociation cross sections, Raman scattering cross sections, etc.) from such wave packet trajectories, and successfully applied their formalisms to a number of experimentally relevant systems. Nevertheless, the wave packet evolution predicted by simple Gaussian wave packet dynamical techniques is in general approximate, and, indeed, situations often arise where an initially Gaussian does not remain Gaussian over a time interval long enough to extract the desired state-to-state transition probabilities. ${ }^{4}$ Thus, it would be desirable to have a way to correct the deficiencies of simple Gaussian wave packet propagation algorithms which retains as fully as possible their physical transparency and algorithmic simplicity.

One approach along these lines involves construction of

\footnotetext{
a) NSF Presidential Young Investigator, Alfred P. Sloan Foundation Fellow, Camille and Henry Dreyfus Teacher-Scholar.
}

a complete set of time-dependent Hermite-Gaussian basis functions "on top of " a central Gaussian wave packet. ${ }^{3(\mathrm{c}), 5}$ The superposition coefficients which specify the amplitude of each basis function are also taken to be time dependent, and their evolution is governed by a simply specified set of linearly coupled first-order ordinary differential equations. The success of any such scheme depends on the rapidity of its convergence as the size of the basis set is increased. Initial applications of Gaussian wave packet-based traveling basis sets were modestly successful, ${ }^{3(c), 5}$ but displayed numerical instability when pressed to treat situations where the simple Gaussian wave packet method is qualitatively inapplicable. This suggests inadequacy of the zeroth order basis set, and forces us to seek a more flexible one. We have recently identified such a basis set, ${ }^{6}$ and the purpose of the present paper is to discuss the details of its construction and demonstrate its potential utility for a variety of problems in quantum molecular dynamics.

The starting point of our present efforts is the time-dependent Hartree-grid (TDHG) method. This is an implementation of the well-known Hartree approximation ${ }^{7}$ in which the overall system wave packet is forced to factorize into a product of single-degree of freedom wave packets. The McLachlan variational principle ${ }^{7}$ then prescribes "optimal" evolution of each single degree-of-freedom wave packet. Because the wave packet is not constrained to be Gaussian in each degree of freedom, a TDHG trial function is more flexible than its Gaussian analog, and can accurately represent many of the non-Gaussian distortions typically encountered on potential surfaces associated with molecular scattering and spectroscopic problems. To correct for the neglect of "direction correlation" in factorized Hartree wave packets, we expand the exact wave function as a superposition of Hartree-type basis wave packets: The superposition coefficients are allowed to vary with time, and in analogy to the Hermite-Gaussian basis originally employed, ${ }^{3(c), 5}$ evolve according to simply prescribed linearly coupled first-order dif- 
ferential equations. The details of this algorithm are presented in Sec. II. Section III presents the results of an application of the proposed methodology to collinear scattering of an atom with a Morse oscillator, a problem which has recently been studied within the TDHG approximation. ${ }^{6}$ We find here that the inclusion of configuration interaction dramatically improves our control over the quality of the results. In particular, it becomes easy to propagate extended non-Gaussian initial wave packets which arise in wave packet theory of transition probabilities for systems possessing initial vibrational excitation. Section IV then summarizes the strengths and weaknesses of our algorithm, compares it with a very recently proposed and related method of Meyer et al., ${ }^{8}$ and suggests further extensions and applications of the ideas developed here.

\section{THEORETICAL BACKGROUND}

For a diatomic molecule colliding with an atom in a collinear fashion the total Hamiltonian can be written as ${ }^{11}$

$$
H=T_{x}+v_{x}(x)+T_{y}+v_{y}(y)+V_{I}(x, y),
$$

where $T_{x}$ and $T_{y}$ correspond to the kinetic parts of a single degree of freedom for $x$ and $y$ coordinates, e.g. [with $\hbar=1$ throughout $] T_{x}=\left(-1 / 2 m_{x}\right) \partial_{x}^{2} \cdot v_{x}(x)$ is the "single particle", or more properly, the single degree of freedom potential associated with coordinate $x ; v_{y}$ is defined analogously. It is important to appreciate that $v_{x}$ and $v_{y}$ can effortlessly assume any functional form in the propagation scheme to be outlined in this section. However, to be specific let us label the free particle coordinate by $y$, so that $v_{y}(y)=0 . x$ is then the diatom vibrational coordinate with $v_{x}(x)$ the potential governing the oscillations of the free (precollision and postcollision) diatom; it is taken to be of Morse form in the numerical application discussed in Sec. III. Finally, $V_{I}(x, y)$ provides the coupling potential which links the motion of coordinates $x$ and $y$. Physically, it is responsible for the vibrational energy transfer induced by the collision; mathematically, it prevents the overall system wave packet $\psi(x, y)$ from factorizing into $x$ and $y$ pieces.

It will prove useful to briefly review the TDHG prescription for computing collisional transition probabilities, since it inspires the extension of the method to include "configuration interaction". In a wave packet formulation the $i$ th column of the $S$-matrix, which contains the transition amplitudes from initial internal (here, vibrational) state $i$ to any final internal state $f$, can be obtained by propagating an appropriate two-dimensional wave packet $\psi(x, y, t)$ into and then out of the scattering region (defined as the region of $y$ coordinate space where $V_{l}$ is nonzero). Specifically, one chooses $\psi(x, y, 0)$ to be incident upon the scattering center, and to factorize as $\chi_{i}(x) G(y)$, where $\chi_{i}$ is the vibrational eigenfunction associated with the initial vibrational state, and $G$ is an incoming translational wave packet. The precise form of $G$ is actually arbitrary, in the sense that exact propagation should yield the same $S$-matrix elements for any $G(y)$, but in practice it is prudent to choose a packet which focuses into the interaction region in order to minimize spatial extension in this region. ${ }^{3(a)}$ From the asymptotic exiting wave packet $\psi(x, y, \tau)$ [where $\tau$ denotes any asymptotically long time], one extracts ${ }^{3(a)}$

$$
\left|S_{f, i}(E)\right|^{2}=\left|B_{f}(E) / A_{i}(E)\right|^{2},
$$

with

and

$$
\begin{aligned}
B_{f}(E)= & {\left[k_{f}(E)\right]^{-1 / 2} \int d x d y \exp \left[-i k_{f}(E) y\right] } \\
& \times \chi_{f}(x) \psi(x, y, \tau)
\end{aligned}
$$

$$
A_{i}(E)=\left[k_{i}(E)\right]^{-1 / 2} \int d y \exp \left[i k_{i}(E) y\right] G(y) .
$$

[Integrals are over all space unless otherwise indicated.] In these expressions $k_{i}$ and $k_{f}$ are pre- and post-collision wave vectors determined from conservation of total energy $E$ according to $k_{i}^{2} / 2 m_{y}+\epsilon_{i}=k_{f}^{2} / 2 m_{y}+\epsilon_{f}$, where $\epsilon_{i, f}$ is the energy eigenvalue associated with vibrational eigenstate $\chi_{i, f}$.

It is natural in this context to consider a TDH ansatz, in which the system wave packet is approximated as

$$
\psi(x, y, t)=\exp [i S(t)] \varphi_{x}(x, t) \varphi_{y}(y, t),
$$

with $S(t)$ a position independent phase angle [not to be confused with $S$-matrix element $\left.S_{f i}(E)\right]$ explicitly isolated in order to simplify the equations of motion for the wave packets $\varphi_{x}$ and $\varphi_{y}$. Specifically, appeal to the McLachlan variational yields well-known mean field equations in which $\varphi_{x}$ obeys a single degree of freedom Schrodinger equation with the effect of the $y$ motion "felt" through a time-dependent effective potential: ${ }^{7}$

$$
\begin{aligned}
i \partial_{l} \varphi_{x}(x, t)= & {\left[T_{x}+v_{x}(x)+\int d y\left|\varphi_{y}(y, t)\right|^{2} V_{l}(x, y)\right] } \\
& \times \varphi_{x}(x, t) .
\end{aligned}
$$

An analogous equation governs the evolution of $\varphi_{y}$, and $S(t)$ depends on the average interaction potential according to $S(t)=\int_{0}^{t} d t^{\prime}\left\langle V_{I}\left(t^{\prime}\right)\right\rangle$, where

$$
\left\langle V_{l}(t)\right\rangle=\int d x d y\left|\varphi_{x}(x, t) \varphi_{y}(y, t)\right|^{2} V_{I}(x, y) .
$$

This prescription has several appealing features. First and foremost, it greatly simplifies the complexity of the partial differential equations which must be integrated numerically. In the present problem, one two-dimensional Schrodinger equation has been replaced by two one-dimensional Schrodinger equations (which must be integrated in tandem due to their mean-field type influence on each other). In general, to solve a $D$-dimensional Schrodinger equation requires representation on a $D$-dimensional spatial grid consisting of $N^{D}$ points, where $N$ is the number of points needed to span each dimension (typically,we find in numerical applications that $N=100-300$ ). In contrast, to solve a $D$-dimensional Schrodinger equation with the TDH approximation requires $N D$ points. Thus the computational effort required to implement the TDH approximation scales roughly linearly with dimensionality, whereas the corresponding effort needed to integrate the Schrodinger equation exactly scales exponentially with dimensionality. A second useful feature of the TDHG algorithm is that all relevant integrals arising in the extrac- 
tion of asymptotic state projections [cf. Eq. (2.2)] factor into products of one dimensional integrals.

Of course, these advantages are moot if the TDHG method does not provide a reasonable approximation to the solution of the appropriate time-dependent Schrodinger equation. Precisely how good is "good enough" depends on the application at hand. For monitoring coarse-grained properties such as the average energy in a particular vibrational mode, the TDH approximation has been shown to be reasonable in several cases. ${ }^{9}$ Extraction of individual stateto-state transition probabilities is a more delicate task. Recent applications indicate the essential promise of the method, ${ }^{6,10}$ but there is clearly room for improved control over the quality of the results. A natural extension would allow us to build upon the qualitative sensibility of TDHG results in order to obtain quantitative accuracy in extracted observables. One obvious class of candidates would be a superposition of linearly independent TDH-type wave packets with coefficients that can be varied to enable "interaction" between basis wave packets or, in the language of electronic structure theory, "configurations." We develop a particularly streamlined version of this idea in the next paragraphs.

For concreteness, let us assume the initial wave packet is Gaussian-like, for example $\psi(x, y, 0)=\chi_{0}(x) G(y)$, where $G(y)$ is a Gaussian wave packet incident upon the scattering center and $\chi_{0}$ the Morse oscillator vibrational eigenfunction. Notice that it is not necessary that the wave packets be strictly Gaussian. It will become clear as the development unfolds that they do not have to be nodeless, either, but for simplicity we consider this case first. It is easy to construct a complete orthonormal set of initial "excited state" basis functions $\varphi_{x}^{(j)}(x, 0) \varphi_{y}^{(k)}(y, 0)$, where $\varphi_{x}^{(j)}(x, 0)=\chi_{j}$, and $\varphi_{y}^{(k)}(y, 0)$ is the $k$ th member of an orthonormal traveling HermiteGaussian basis set. To be specific about $\varphi_{y}^{(k)}$ we use Heller's notation for a general Gaussian wave packet, ${ }^{1(a)}$ i.e., take

$$
G(y)=\exp \left\{i\left[\alpha_{0}\left(y-y_{0}\right)^{2}+p_{0}\left(y-y_{0}\right)+\gamma_{0}\right]\right\},
$$

where $y_{0}$ is the position-space center of the Gaussian, $p_{0}$ its momentum expectation value, $\alpha_{0}$ contains spread and "contraction" information (a fuller explanation of this point is provided in Sec. III), and $\gamma_{0}$ ensures unit normalization (its real part also provides an overall phase factor, which we can eliminate with no loss of generality by choosing $\operatorname{Re} \gamma_{0}=0$ ). Then the desired initial orthonormal Hermite-Gaussian basis functions are given by ${ }^{5}$

$$
\varphi_{y}^{(k)}(y, 0)=c_{k} H_{k}\left[\left(2 \operatorname{Im} \alpha_{0}\right)^{1 / 2}\left(y-y_{0}\right)\right] G(y),
$$

with $H_{k}$ the $k$ th Hermite polynomial and $c_{k}=\left[2^{k} k !\right]^{-1 / 2}$.

The most obvious configuration interaction scheme would utilize basis functions which have been subjected one by one to the TDHG approximation. However, a problem arises, because each basis wave packet evolves under its own variationally optimized separable effective potential [cf. Eq. (2.3) ]; hence they do not remain orthonormal. This severely encumbers the equations of motion for the basis coefficients which must be determined in order to determine the "configuration interaction." Fortunately, a simple remedy exists, namely to propagate all basis under the same separable, effective potential. Several possibilities are immediately suggested, the most obvious being the TDH effective potential associated with the initial ground state basis function $\varphi_{x}^{(0)}(x, 0) \varphi_{y}^{(0)}(y, 0)=\chi_{0}(x) G(y)$. We adopt this choice in the discussion which follows.

Anticipating the central role of the ground state basis wave packet, let us denote it as $\bar{\varphi}_{x}(x, t) \bar{\varphi}_{y}(y, t)$. The simplest way to obtain this packet is to subject the initial packet $\chi_{0}(x) G(y)$ to the mean field Eqs. (2.3a). We then compute and store the time-dependent one-dimensional effective potentials generated thereby, namely

$$
V_{x}^{\mathrm{eff}}(x, t)=v_{x}(x)+\int d y\left|\bar{\varphi}_{y}(y, t)\right|^{2} V_{I}(x, y)
$$

and analogously for $V_{y}^{\mathrm{eff}}(y, t)$. Again, we emphasize that $\bar{\varphi}_{x}$ is obtained by propagating $\varphi_{x}^{(0)}(x, 0)$ according to the potential in Eq. (2.4) self-consistently with its $\bar{\varphi}_{y}$ counterpart. All the other $x$ basis wave packets $\varphi_{x}^{(k)}(x, t), k>0$, are then obtained by propagating $\varphi_{x}^{(k)}(x, 0)$ according to $V_{x}^{\text {eff }}(x, t)$ in Eq. (2.4), and likewise for the other $y$ basis wave packets. Now, we consider a superposition of basis functions generated in this way

$$
\psi(x, y, t)=\sum_{j, k} a_{j k}(t) \theta_{j k}(x, y, t),
$$

where

$$
\theta_{j k}(x, y, t) \equiv \exp [i S(t)] \varphi_{x}^{(j)}(x, t) \varphi_{y}^{(k)}(y, t),
$$

and $S(t)$ is the phase angle $S(t)=\int_{0}^{t} d t^{\prime}\left\langle V_{I}\left(t^{\prime}\right)\right\rangle$ based on the average interaction potential

$$
\left\langle V_{I}(t)\right\rangle=\int d x d y\left|\bar{\varphi}_{x}(x, t) \bar{\varphi}(y, t)\right|^{2} V_{I}(x, y) .
$$

In Eq. (2.5) $a_{j k}$ are time-dependent coefficients whose equations of motion are greatly simplified by two important (and related) features of the adopted basis set. First, the basis functions remain orthonormal for all times, i.e.

$$
\begin{aligned}
& \left\langle\theta_{j k}(x, y, t) \mid \theta_{j^{\prime} k^{\prime}}(x, y, t)\right\rangle \\
& \quad=\left\langle\varphi_{x}^{(j)}(x, t) \varphi_{y}^{(k)}(y, t) \mid \varphi_{x}^{(j)}(x, t) \varphi_{y}^{\left(k^{\prime}\right)}(y, t)\right\rangle \\
& \quad=\delta_{j j^{\prime}} \delta_{k k^{\prime}} .
\end{aligned}
$$

This property follows in essence from the second useful feature of these basis functions, namely that all correspond to evolution under the same time-dependent Hermitian Hamiltonian. Specifically,

$$
\begin{aligned}
i \partial_{t} \theta_{j k}(x, y, t)= & {\left[T_{x}+T_{y}+V_{x}^{\mathrm{eff}}(x, t)+V_{y}^{\mathrm{eff}}(y, t)\right.} \\
& \left.-\left\langle V_{I}(t)\right\rangle\right] \theta_{j k}(x, y, t),
\end{aligned}
$$

with

$$
\left\langle V_{I}(t)\right\rangle=\left\langle\bar{\varphi}_{x}(x, t) \bar{\varphi}_{y}(y, t)\left|V_{I}(x, y)\right| \bar{\varphi}_{x}(x, t) \bar{\varphi}_{y}(y, t)\right\rangle .
$$

Now, when the trial function in Eq. (2.5) is substituted into the full time-dependent Schrodinger equation, properties $(2.6,2.7)$ can be utilized to show that the trial function solves the Schrodinger equation if the coefficients $a_{j k}$ evolve according to

$$
i \dot{a}_{j k}(t)=\sum_{j, k^{\prime}} \mathscr{H}(t)_{j k^{\prime} k^{\prime}} a_{j^{\prime} k^{\prime}}(t),
$$

where the coupling coefficients $\mathscr{H}(t)_{j k^{\prime} k^{\prime}}$ correct for the error in using an approximate separable potential to propagate the basis wave packets. Specifically, 


$$
\begin{aligned}
\mathscr{H}(t)_{j k j^{\prime} k^{\prime}}= & \left\langle\varphi_{x}^{(j)}(x, t) \varphi_{y}^{(k)}(y, t)\right| \Delta V_{I}(x, y, t) \\
& \times\left|\varphi_{x}^{(j)}(x, t) \varphi_{y}^{\left(k^{\prime}\right)}(y, t)\right\rangle,
\end{aligned}
$$

with

$$
\begin{aligned}
\Delta V_{I} \equiv & V_{I}(x, y)-\left[\int d y\left|\bar{\varphi}_{y}(y, t)\right|^{2} V_{I}(x, y)\right. \\
& \left.+\int d x\left|\bar{\varphi}_{x}(x, t)\right|^{2} V_{I}(x, y)-\left\langle V_{I}(t)\right\rangle\right] .
\end{aligned}
$$

The content of Eqs. (2.8) may be summarized in matrix notation as $i \ddot{a}(t)=\mathscr{H}(t) \mathbf{a}(t)$, in which it is understood that the coefficients $a_{j k}(t)$ are arranged into an array in some convenient manner and $\mathscr{H}(t)$ contains the matrix elements $\mathscr{H}(t)_{j k, j, k}$ appropriately organized.

If the superposition amplitudes of basis components is determined in the manner just described, $\psi(x, y, t)$ becomes formally exact as the number of basis functions is increased ad infinitum. Of course, it remains to be shown that convergence can be obtained with a modest number of basis functions (say, 3-10 per degree of freedom, although this number may vary considerably from application to application). Section III below illustrates the ease with which the method handles one nontrivial example. Before proceeding with specific numerical results, we summarize some of the expected advantages of the formulation we have developed here.

First, the prescription is physically motivated in that it builds directly upon the TDH-level approximation, which should be qualitatively reasonable in many situations. The only aspect of the dynamics which is improperly described then is the "direct correlation" of the motion in various degrees of freedom, or more precisely, the nonfactorizability of the exact multidimensional wave packet. This manifests itself geometrically as a twisting of the wave packet away from the $x$ - $y$ axes (cf. Sec. III). It is precisely this effect which the "configuration interaction" (CI) is designed to handle (by superimposing several linearly independent factorized basis functions). As long as the central Hartree basis wave packet locates the important region of configuration space (or, more properly, phase space, since the "momentum wiggles" in the wave function must be accounted for, too), we have a reasonable expectation of pinning down the details of its spatial behavior via $\mathrm{CI}$ corrections.

Technically, the CI method introduced here has a number of attractive features. First, the effective potential used to propagate the basis functions needs to be determined only once from a simple TDHG integration which itself scales essentially linearly with the spatial dimensionality of the system. Subsequent to this, each basis function is evolved in a known one-dimensional potential. Thus its integration is completely uncoupled from the integration of the other basis functions. As a result, the computational effort needed to determine the time-dependent basis functions is expected to scale in a strictly linear fashion with spatial dimensionality.

Another advantage of the prescription is that it is an interaction picture formulation in which the zeroth order Hamiltonian corresponds to motion on the effective separable potential specified above. In particular, if the potential experienced by the moving wave packet becomes separable after some period of time, $\Delta V_{I} \rightarrow 0$ and the basis coefficients reach asymptotic values. In practice, this should prove very useful in direct scattering and photodissociation applications, where configuration interaction has to be performed only for the short time that the wave packet is in the interaction region.

A third virtue of our scheme is the opportunity afforded for factorization of various required integrations into one dimensional components. Clearly, projections upon asymptotic eigenfunctions are easily performed, since the TDHtype basis functions naturally factor in the asymptotic coordinates $x$ and $y$. Furthermore, it should usually be possible to express the interaction potential $V_{I}$ as the sum of a small number of functions which also factorize. In this case all the matrix elements needed to compute the CI coupling matrix $\mathscr{H}(t)$ factor into products of one dimensional integrals.

\section{APPLICATION TO COLLINEAR ATOM-MORSE OSCILLATOR SCATTERING}

In order to demonstrate the practical aspects of our CI formalism, we adopt a model for collinear He plus Morse oscillator $\mathrm{H}_{2}$ scattering which was recently used to study the applicability of the TDHG method for inelastic molecular scattering processes. ${ }^{6}$ The potential involved is of modified Secrest-Johnson (SJ) -type, ${ }^{11}$ i.e., in the notation of the previous section

$$
\begin{aligned}
& V_{x}(x)=D[1-\exp (-\beta x)]^{2} \\
& V_{I}(x, y)=\exp [-\alpha(y-x)] .
\end{aligned}
$$

As in the SJ potential, we choose the inverse length scale of the exponentially repulsive interaction potential to have the value of $\alpha=0.3$. We then replace the unit force constant harmonic potential studied by SJ with a strongly anharmonic Morse potential having the same curvature at its equilibrium point, but containing only 40 bound states. This is done by choosing $D=20.0$ and $\beta=0.158$. (Remember that $\hbar=1$.) With the choice of masses (following SJ) $m_{x}=1, m_{y}=2 / 3$, the scattering problem is completely specified. Complete solution of this collision problem (for all initial vibrational states $i$ and all energies $E$ ) by simple approximate wave packet dynamical techniques (e.g., Gaus$\operatorname{sian}^{4(b), 4(c)}$ and TDHG $^{6}$ ) has proven elusive. Consequently, the ease with which it yields to our TDHG-CI formalism will serve to establish the method's potential.

All of our calculations begin with the isolation of an appropriate "ground state" TDHG wave packet trajectory to be used as the central trajectory for determining $S$-matrix elements over a modest range of energies. Typically, by adjusting the focus of the incident translational wave packet [essentially, by varying $\operatorname{Re} \alpha_{0}$ in $G(y)$ ] it was straightforward to find a Hartree trajectory which gave reasonably stable probabilities for transitions out of the $i=0$ incident vibrational state over the range of energies contained in the incident packet. (Stability is reckoned by computing $\Sigma_{f}\left|S_{f, 0}(E)\right|^{2}$, where the sum is over all energetically open final state channels, and should in principle be one for all energies. Typically, it is not hard to find a Hartree trajectory which satisfies this unitarity check to within a few percent 
over 5 or more units of energy.) Once an acceptable central Hartree trajectory has been identified, $\mathrm{CI}$ corrections can be implemented in the manner detailed in the previous section.

The Hartree approximation is known to work well for extracting $0 \rightarrow f$ transition probabilities in inelastic scattering problems of this type. ${ }^{6}$ Hartree-based results for energies $E=10,12$, and 14 are indicated by the open circles in Figs. 1 $(\mathrm{a}, \mathrm{b}$, and $\mathrm{c})$, respectively. All were obtained from the same TDHG wave packet trajectory. By employing the CI scheme described in Sec. II with a basis that consists of all products of functions having $j, k=0-4$, these results are modified slightly, as indicated by the open squares in Fig. 1. (For all calculations presented in this section, we have employed the same number of basis functions $M$ in both coordinates, e.g., $M=5$ for the $\mathrm{CI}$ results displayed in Fig. 1. However, there is no reason in general why basis functions cannot be concentrated more heavily on the "difficult" degrees of freedom.) Exact results, obtained by discretizing the wave packet on a two dimensional grid and propagating it according to a standard FFT split-operator algorithm, ${ }^{12(a)}$ are indistinguishable from the $M=5 \mathrm{CI}$ results on the scale of this figure. [In addition, the results reported in this paper (except for $E=22$, below) were checked via a standard time-independent coupled channel scattering code. $\left.{ }^{12(b)}\right]$ Through this agreement, we gain confidence in the CI formalism developed in Sec. II.

In subsequent paragraphs we continue to explore the accuracy and flexibility of our CI algorithm, but first we pause briefly to examine the wave packet trajectory which gives rise to the transition probabilities indicated in Fig. 1. Figure 2 shows snapshots of the probability density of the exact (fully coupled) wave packet. Note that the exact wave packet does not twist, i.e., it remains oriented along the $x-y$ axes at all times. This means that a TDHG wave packet has the flexibility to follow the exact motion in this case. Indeed, comparison of the TDHG trajectory used to obtain the results shown in Fig. 1 and the exact wave packet density reveals that the two are indistinguishable on the scale of resolution of Fig. 2. This observation is consistent with the high accuracy of $S$-matrix information extracted from the TDHG wave packet trajectory.

We now consider systems initially prepared in an excited oscillator state $i>0$. As was observed in Ref. 6, direct application of the TDHG method fails for these more extended states. The problem was partially circumvented in Ref. 6 by decomposing the excited oscillator wave packet into several localized Gaussian pieces. Each of these was propagated independently under the TDHG approximation, and then the resulting wave packet trajectories added coherently to produce an approximation to the exact excited state wave packet trajectory. $S$-matrix results obtained by this procedure were qualitatively satisfactory, although the quantitative agreement with the exact results was not so good as in the $i=0$ case. Here we find that utilization of our CI correction algorithm enables us to completely avoid these difficulties and obtain accurate $S$-matrix elements with the same level of effort needed to obtain $i=0$ results.

The scheme proceeds in the same way as for the $i=0$ case. One chooses a reasonable Gaussian-type TDHG tra-

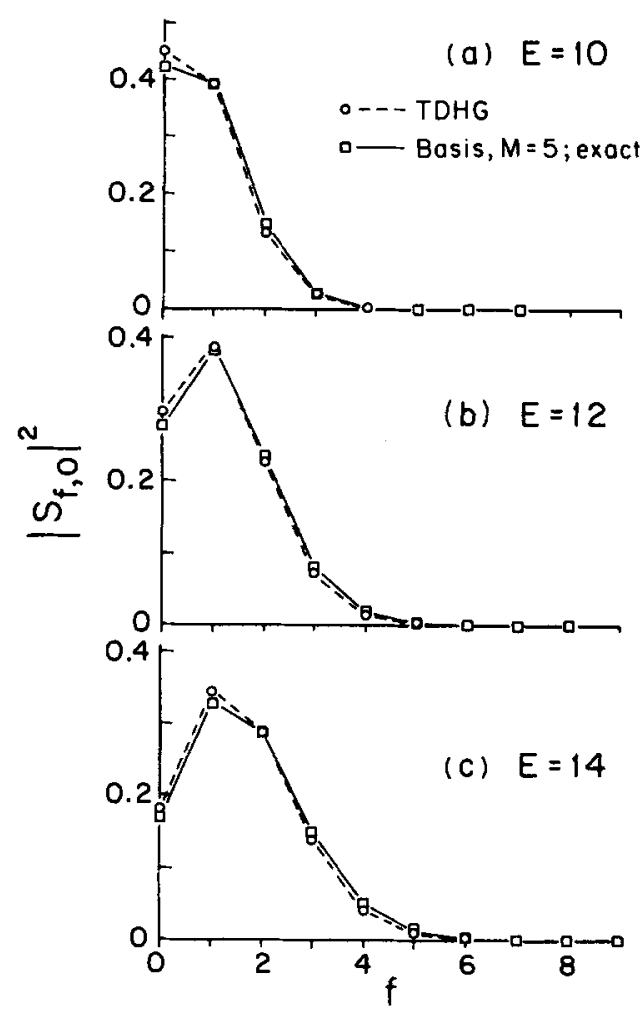

FIG. 1. Panels (a), (b), and (c) show $0 \rightarrow f$ vibrational transition probabilities at energies $E=10,12$, and 14 , respectively, for the inelastic scattering model adopted in the text. TDHG results are indicated by open circles, $M=5$ basis and exact 2-D grid integrator results by open squares.

jectory to "anchor" the traveling basis set. [Essentially, the center of momentum $p_{0}$ in the incident Gaussian $G(y)$ is chosen such that $p_{0}^{2} / 2 m_{y}+\epsilon_{i} \cong E$, where $i$ is the initial vibrational state for which $S$-matrix elements are desired, and $E$ is a typical energy at which the $S$-matrix is to be comput-

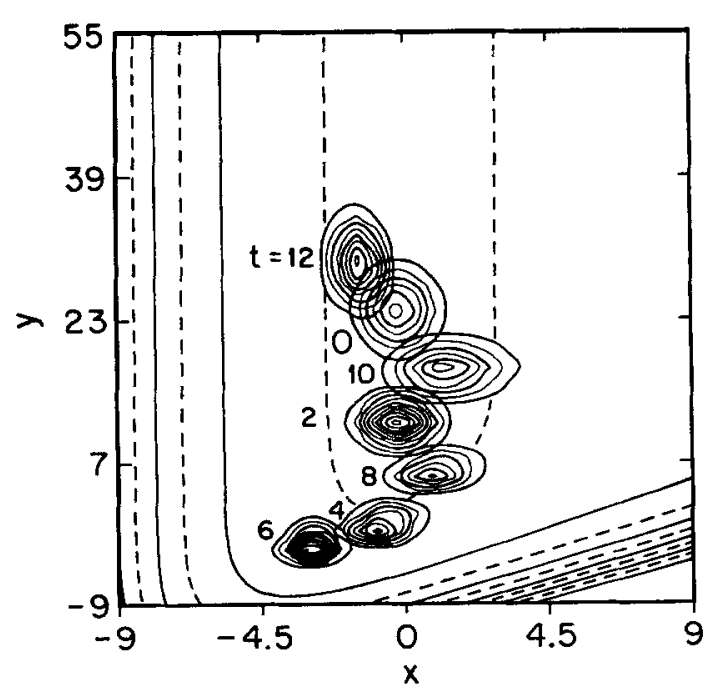

FIG. 2. Contour plots of exact wave packet probability density for $i=c$ scattering wave packet at indicated times. 


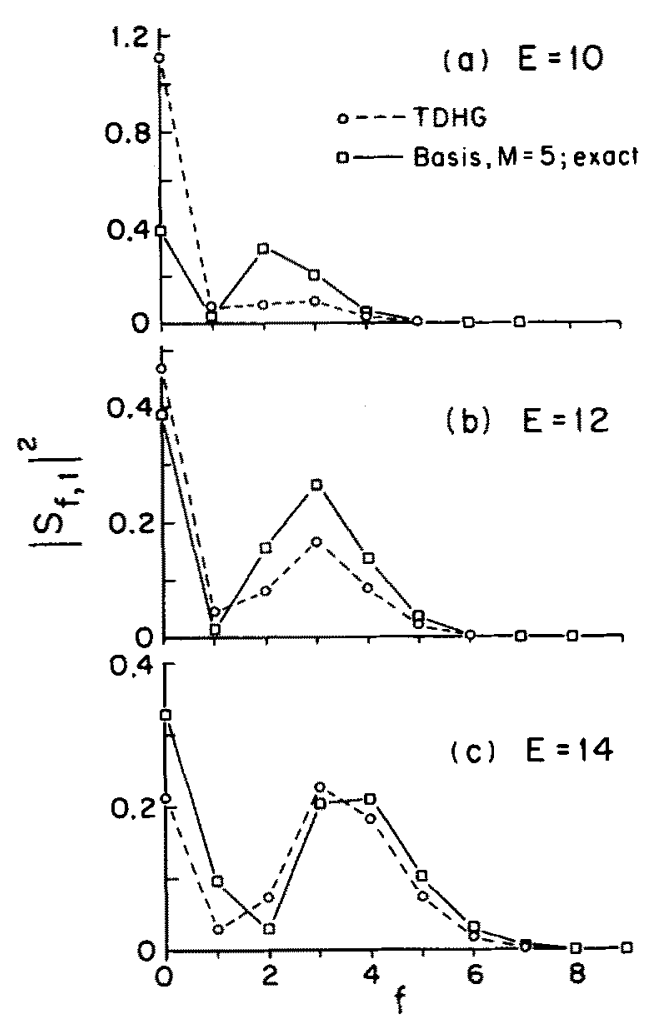

FIG. 3. Panels (a), (b), and (c) show $1 \rightarrow f$ vibrational transition probabilities at energies $E=10,12$, and 14 , respectively, for the inelastic scattering model adopted in the text. TDHG results are indicated by open circles, $\boldsymbol{M}=5$ basis and exact 2-D grid integrator results by open squares.

ed. ] For the configuration interaction phase of the computation [cf. Eq. (2.8)], the appropriate initial condition in general is $a_{i 0}(0)=1$, and all the other $a_{j k}(0)=0$. Results for energies $E=10,12$, and 14 are shown in panels (a), (b), and (c) of Fig. 3 for the case $i=1$ and Fig. 4 for the case $i=2$. For $i=1$, a $\mathrm{CI}$ basis characterized by $M=5$ is sufficient to obtain results which are in quantitative agreement with the exact answers. For $i=2$, it is necessary to go to a slightly larger basis size, namely $M=8$, to get complete agreement with the exact results, but for the most part the errors are minor even when $M=5$. These $\mathrm{CI}$ results for initial wave packet states associated with $i=1$ and 2 represent a significant improvement over those obtained at the simple TDHG level (i.e., propagation of $\chi_{i} G(y)$ directly via the TDHG approximation). The simple TDHG results are shown via open circles in each panel of Figs. 3 and 4, and are seen to be of rather poor quality.

It is interesting to compare the evolving probability densities associated with TDHG, $\mathrm{CI}$ and exact wave packet trajectories for a system of initially prepared in an extended wave packet state. As an example, we show in Fig. 5(a) the TDHG result associated with $i=2$, while Fig. $5(\mathrm{~b})$ contains the behavior of the corresponding exact wave packet. The exact wave packet probability distorts appreciably when it impacts the repulsive barrier of the potential energy surface. In particular, it twists noticeably (e.g., at times $t=6$ and 12). This effect cannot be accommodated by a single TDHG

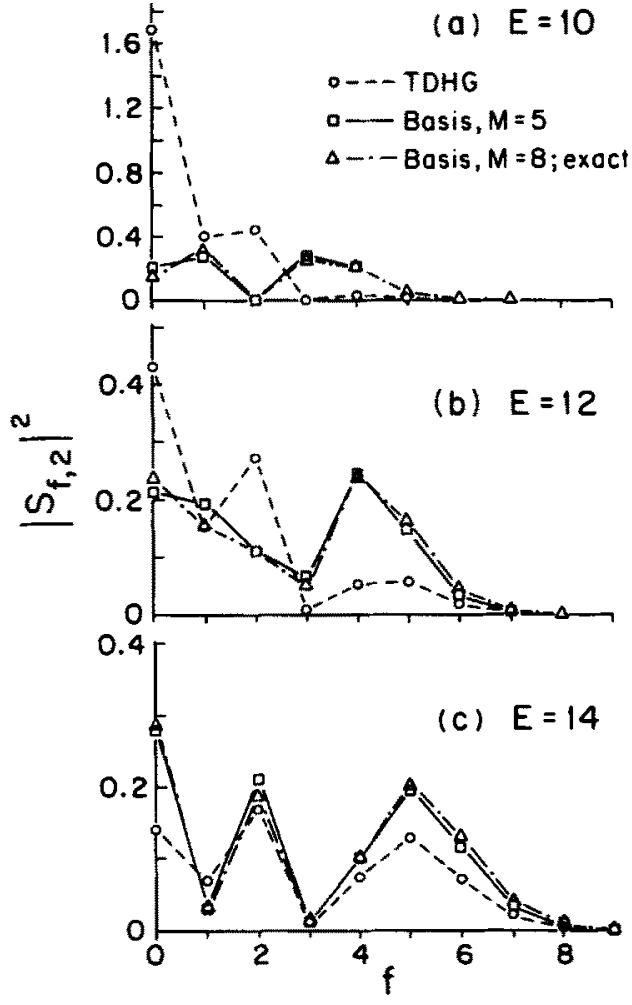

FIG. 4. Panels (a), (b), and (c) show $2 \rightarrow f$ vibrational transition probabilities at energies $E=10,12$, and 14, respectively, for the inelastic scattering model adopted in the text. TDHG results are indicated by open circles, $M=5$ basis results by open squares, and $M=8$ basis and exact $2-\mathrm{D}$ grid integrator results by open triangles.

wave packet. As is clear from Fig. 5(a), a TDHG packet must always remain rigidly oriented parallel to the $x-y$ axes. These pictures clearly illustrate the concept of "direct correlation" is much more substantial for spatiality extended wave packets such as those which arise in the study of collisions involving vibrationally excited initial states. A simple argument for the degradation of the TDHG approximation with the spatial extent of the propagating wave packet runs as follows: In a sufficiently local region about some point $\left(x_{t}, y_{t}\right)$ the interaction potential separates according to

$$
\begin{aligned}
V_{I}(x, y) \cong & V_{I}\left(x_{t}, y_{t}\right)+\partial_{x} V_{I}\left(x_{t}, y_{t}\right)\left(x-x_{t}\right) \\
& +\partial_{y} V_{I}\left(x_{t}, y_{t}\right)\left(y-y_{t}\right) .
\end{aligned}
$$

[A separable potential closer to the spirit of the TDHG approximation can be constructed, as discussed in the next section, but the decomposition just indicated suffices for present purposes.] An initially factorized wave packet will remain factorized until this approximation breaks down. Obviously, the more spatially localized the packet, the longer factorization is expected to persist, so the behavior of the exact wave packet when $i=0$ (Fig. 2) vs $i=2[$ Fig. $5(\mathrm{~b})]$ is not surprising. (The presence of nodes in the evolving wave function may also contribute to the breakdown in accuracy of the TDHG method, but we have not explored this possibility in any detail.) An important point here is that for $M \gtrsim 5$ our $\mathrm{Cl}$ scheme produces snapshots which are virtually 

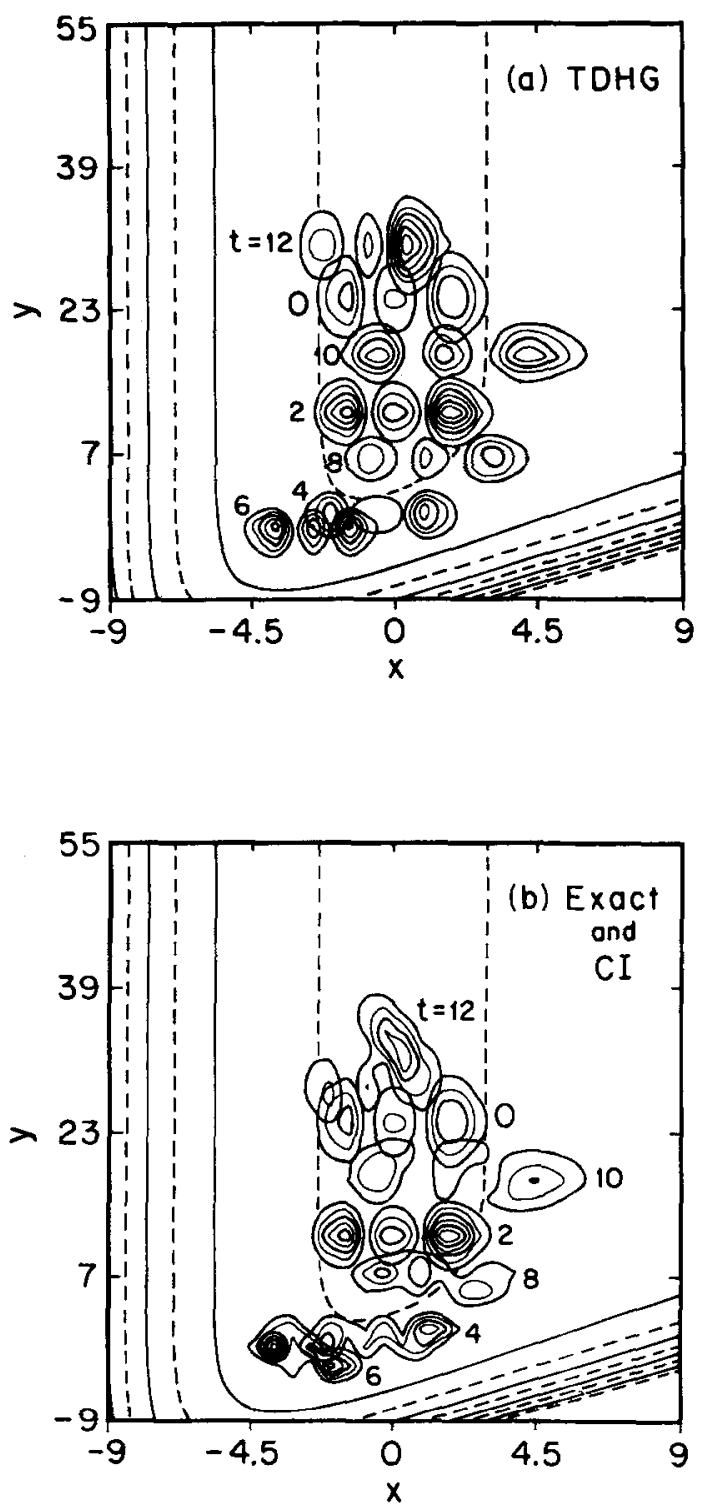

FIG. 5. Contour plots of wave packet probability density for $i=2$ scattering wave packet at indicated times. Panel (a) shows the TDHG approximant, while panel (b) shows the $M>5$ basis and exact 2-D grid integrator results.

indistinguishable from the exact $i=2$ wave packet [Fig. 5 (b) ]. This is another indication that the method can naturally and efficiently account for correlation effects. It is $a b$ initio, and provided its computational feasibility scales favorably with spatial dimension, may prove useful in a variety of applications.

As a final demonstration of the utility of our method we employ it to extract $S$-matrix elements for the energy $E=22$. This energy is above the dissociation threshold of the Morse oscillator. A continuum of unbound Morse oscillator eigenfunctions is now energetically accessible, and the need to include these functions in any straightforward timeindependent basis expansion requires substantial modification of standard coupled channel codes. Time-dependent wave packet methods are considerably less sensitive to the opening of "reactive channels." Indeed, for the collinear $\mathrm{H}_{2}-$ He scattering application under study here (in which the "reactive channel" involves dissociation of the $\mathrm{H}_{2}$ molecule), both the TDHG method and the CI scheme built upon it are impervious to such complications in the energy range examined (i.e., relatively near the dissociation threshold).

Implementation of these procedures proceeds exactly as in the low-energy (i.e., predissociation) cases considered above. First, a suitable TDHG trajectory must be isolated. We used the one associated with Fig. 1 of Ref. 6, which produces the $0 \rightarrow f$ transition probabilities indicated by the open circles in Fig. 6(a). CI corrections to these results were then computed using the algorithm introduced in Sec. III. Converged $0 \rightarrow f$ transition probabilities (obtained with $M=5$ ) are shown via the open squares in Fig. 6(a). The same basis wave packets were used in a CI computation of $1 \rightarrow f$ and $2 \rightarrow f$ transition probabilities, with results shown in Figs. 6(b) and 6(c), respectively. For $i=1$, converged results were obtained with $M=5$. This size basis produced nearly converged $2 \rightarrow f$ basis probabilities as well. By increasing the basis set size slightly, the $M=8$, the completely converged results indicated by the open triangles in Fig. 6(c) were obtained. For the sake of comparison, TDHG trajectories were also computed for $i=1$ and $i=2$ [i.e., the TDHG approximation was employed for the initial wave packets $\chi_{i} G(y)$, with the translational Gaussian $G(y)$ cho-

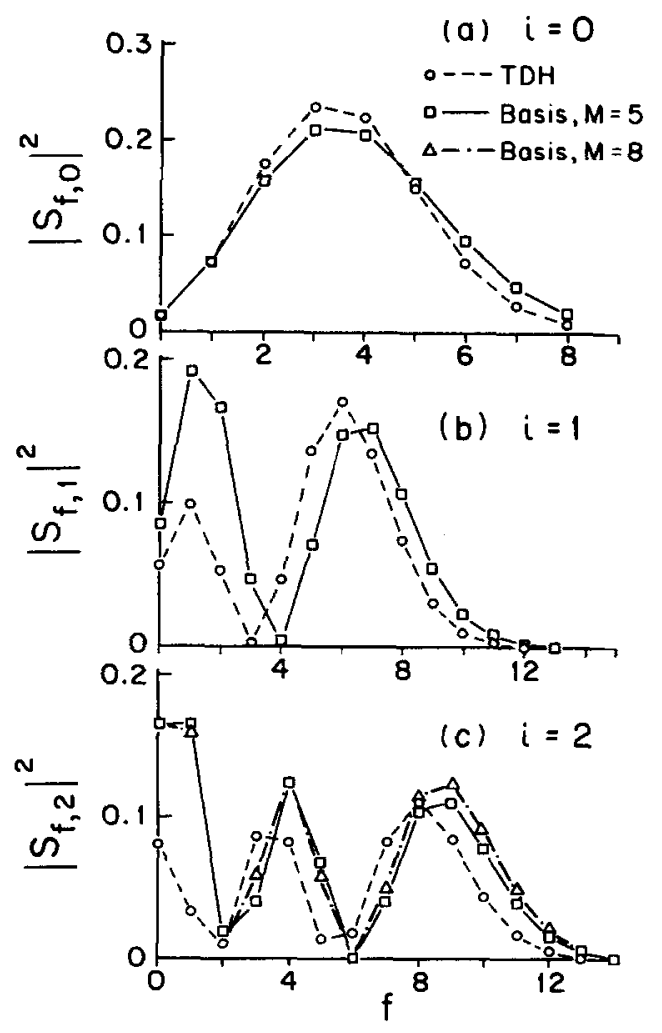

FIG. 6. $i \rightarrow f$ transition probabilities at energy $E=22$ for the inelastic scattering model adopted in the text. Results for $i=0,1$, and 2 are shown in panels (a), (b), and (c), respectively. TDHG results are indicated by open dots, $M=5$ basis results by open squares, and $M=8$ basis results by open triangles. 
sen approximately for energy $E=22]$. Transition probabilities extracted from these trajectories are shown with open circles in Figs. 6(b) and 6(c), respectively. The overall trends are seen to be in agreement with the findings of Ref. 6. For $i=0$, the TDHG-based transition probabilities are reasonably accurate, but this accuracy erodes significantly for the extended initial wave packet states $i=1$ and $i=2$.

We conclude this section with brief comments on the efficiency of the various exact solution techniques which can be utilized in the collision problem under study. Two dimensional grid integrators such as the split-operator FFT meth$\operatorname{od}^{12(a)}$ used here are flexible and reliable for problems involving two spatial dimensions, and so they are routinely used on currently available supercomputers. However, extensions to three and higher dimensions are much more demanding computationally (the growth of computational effort with spatial dimensionality is exponential for this class of methods). Three dimensions is perhaps barely within reach, but four dimensions is currently out of the question. The other class of traditional methods which are commonly used for quantum collision problems are time independent coupled-channel algorithms. ${ }^{12(b)}$ These also slow down exponentially as the spatial dimensionality of the problem increases [and, in addition, require substantial modification as new arrangement channels become energetically accessible (a problem to which time-dependent wave packet methods are much less sensitive)]. It is hoped that computational labor associated with the TDHG-CI method introduced in Sec. II and demonstrated in the present section will scale in a milder way with spatial dimensionality. Already in two dimensions we find a factor of 5 increase in speed relative to our 2-D grid integration codes. More importantly, parts of the TDHG-CI algorithm grow linearly with spatial dimensionality, while other parts, whose growth must formally be classified as "exponential", in practice have simplifying features (extreme sparseness of critical matrices and/or vectors, flexibility with respect to distribution of basis functions on various degrees of freedom, etc.) as discussed in the next section.

\section{DISCUSSION}

Localized wave packet dynamics has been recognized for some time as an appealing way to compute quantum observables. It brings a manifestly dynamical point of view to the subject of quantum dynamics, which is intuitively valuable. It also has computational advantages over traditional time independent approaches for certain classes of problems, for example dissociation or rearrangement events in which multiple continua are energetically accessible. Above all, from a computational point of view, simple localized wave packet methods such as Gaussian wave packet dynamics and the TDHG method are easily extendable to high-dimensional systems. However, these types of methods have been plagued by questionable reliability in many cases. ${ }^{4(b)}$ What is needed is a way to monitor their accuracy and correct for their errors. The algorithm developed in this work, a natural scheme for incorporating coupling between different members of a TDHG level basis, addresses this need directly. It has a number of commendable features, and can be extended and applied in several interesting ways which merit comment here. First we review some of its technical assets;

(i) The construction of the traveling basis functions is exceptionally easy, and scales strictly linearly with spatial dimensionality.

(ii) Since the basis functions are naturally orthonormal at all times, the linearly coupled evolution equations for the time-dependent superposition coefficients have a very simple structure. The coupling matrix $\mathscr{H}$ is also very easy to determine because the basis functions factorize. Provided also that the interaction potential can be broken into a sum of product functions, $\mathscr{H}$ can be constructed as a direct product of 1-D matrices, each of which involve matrix elements of a particular coordinate.

(iii) Asymptotic overlaps factor into products of 1-D integrals as do time-correlation functions typically encountered in electronic absorption and Raman scattering problems. $^{3(e)}$

There are several other intriguing features of the algorithm which have yet to be explored. For example, we find that the coefficient vector $\mathbf{a}(t)$ is usually quite sparse, i.e., only a few of the coefficients are substantially nonzero at any time. To evolve over a small interval, it should be sufficient to examine the coefficient list at the beginning of the interval and compute only those columns of $\mathscr{H}$ which are associated with the nonzero coefficients. In this way it is hoped that the amount of computational labor involved in evolving the supercoefficients $\mathbf{a}(t)$ can be substantially reduced.

One also has a great deal of flexibility in choosing the guiding trajectory upon which the traveling orthonormal basis set it built. We have focused here on a simple possibility, namely utilization of the time-dependent effective separable potential generated for a Gaussian-like $i=0$ initial wave packet according to the TDHG approximation. To make this notion more precise, let us consider the prototypical case that $V_{I}(x, y)=f(x) g(y)$. Then the effective separable interaction potential generated by $\chi_{0}(x) G(y)$ $=\varphi_{x}^{(0)}(x, 0) \varphi_{y}^{(0)}(y, 0)$ is

$$
\begin{aligned}
V_{I}(x, y) \cong & \langle g(y)\rangle_{0} f(x)+\langle f(x)\rangle_{0} g(y) \\
& -\langle f(x)\rangle_{0}\langle g(y)\rangle_{0},
\end{aligned}
$$

where $\langle g(y)\rangle_{0}$ means average $g$ over $\left|\varphi_{y}^{(0)}(y, t)\right|^{2}$, etc. There is no reason why we cannot use the effective potential associated with the TDHG trajectory appropriate to $\varphi_{x}^{(j)}(x, 0) \varphi_{y}^{(k)}(y, 0)$ with $j, k \neq 0$. In fact we have also implemented our CI algorithm for initially vibrationally excited systems (i.e., $i=1,2$ ) by using the TDHG trajectory for the relevant (extended) initial state to generate the global effective potential which determines our basis set. We find that there is no substantial advantage to this variation: both implementations yield the same $S$-matrix elements with about the same basis size. We conclude that one can only do "so well" in choosing an effective separable potential. In essence any TDHG approximation gives $V_{I} \cong V_{I}\left(x, y_{t}\right)$ $+V_{I}\left(x_{t}, y\right)-V_{I}\left(x_{t}, y_{t}\right)$, where $\left(x_{t}, y_{t}\right)$ is the coordinate space center of the packet. Different excited state trajectories give slight variants of this result depending on the dispersion of the wave packet's probability density, but our experience has been that all effective potentials generated in this manner are qualitatively consistent with the form just quoted. Nev- 
ertheless, this exploration suggests other possibilities, which may in fact yield substantial improvement.

In particular, an orthonormal basis set can be built upon a nonlinear variational trial function which already has some correlation built into it. For example, as discussed recently by Kucar et al., ${ }^{13}$ one can consider variational trial functions of the form $\exp \left\{\delta_{t} L_{z}\right\} \varphi_{x}(x, t) \varphi_{y}(y, t)$, where $L_{z}$ is the $z$-component of angular momentum and $\delta_{t}$ a real parameter. This leads to norm-conserving Hartree-type Schrodinger equations for $\varphi_{x}$ and $\varphi_{y}$, plus an equation of motion for $\delta_{i}$. Use of such angular momentum generator allows this trial wave packet to "twist", so it may be capable of building in some correlation in a simple "Hartree-esque" way. It is then simple to construct an orthonormal basis of excited-state wave packets "on top of " such a guiding wave packet, and subsequently carry out configuration interaction in order to completely capture the distortions exhibited by the exact wave packet.

There are two other important classes of problems for which our CI method appears reasonably well suited, namely (1) curve crossing problems and (2) rotational motion. Curve crossing, which entails coupling between wave packets on different potential surfaces, is ubiquitous in reduced descriptions of molecular many-body problems (involving electronuclear coupling, multidimensional tunneling, etc.). It is also much more difficult to treat by simple "classically inspired" localized wave packet methods, since passage of a wave packet through a crossing seam in the multidimensional potential surface is an intrinsically nonclassical event (which leads, for example, to leakage of part of the initial wave packet amplitude). It is plausible that a two-surface TDHG approximation (of the type recently investigated by Kotler et al. ${ }^{14}$ ) can be used to anchor an orthornormal spinor-type basis set (i.e., in which each basis function consists of a Hartree-type coordinate space function times an electronic, spin or tunneling base state vector). Again, once this construction has been accomplished, computation of configuration interaction corrections should be straightforward, albeit somewhat more complicated than for a singlesurface motion.

Let us also briefly consider the incorporation of rotational motion into the procedure. The TDH approximation can be applied in a straightforward manner to rotational or other curvilinear coordinates, and there is no reason why the Hartree-based CI scheme set forth in this paper cannot be directly applied in order to correct for errors in the simple Hartree level of description. To take a simple example, consider scattering of a rigid-rotor diatom from a surface, or its photodissociation analog, desorption of a rigid-rotor diatom from a surface by direct excitation to a repulsive excited state. In these problems the center of mass molecular motion is naturally treated via Cartesian wave packets, whereas the orientation of the diatom can be described through a timedependent superposition of spherical harmonics. ${ }^{15}$ At the Hartree level of description the motion of the angular coordinates is given by a simple matrix differential equation of the canonical form $i \dot{\mathbf{b}}=\mathscr{G}(t) \mathbf{b}$, where $\mathbf{b}(t)$ is the vector of spherical harmonic coefficients, and $\mathscr{G}(t)$ the appropriate Hermitian matrix. ${ }^{15}$ Since it is Hermitian it can be used to propagate a variety of initial coefficient vectors, and if the latter are chosen to be orthonormal at $t=0$, they will remain so for all subsequent times. It only remains to decide upon a "natural" set of orthonormal angular basis function. This choice will depend on the application. For example, in the case of molecule-surface scattering from initial angular momentum state $j=0$, the basis vectors would by simply $\mathbf{b}=(1,0, \ldots),(0,1, \ldots)$, etc.; that is, they should be the set of spherical harmonics themselves. In the case of photodesorption from an initial librational state, whose probability density might be envisioned as a polar icecap on the unit sphere, one would represent this density as a superposition of spherical harmonics, and then construct excited states with increasing nodal structure, for example, by Gram-Schmidt orthogonalization. ${ }^{16}$ This procedure only has to be carried out once, after which the basis functions remain orthonormal naturally.

Before concluding, we wish to point to interesting recent work by Meyer $e t a l .^{8}$ which appeared as we were preparing the present report. These authors appeal to the so-called Lagrangian time-dependent variational principle, ${ }^{17}$ and derive equations of motion for a time-dependent superposition of Hartree-type basis functions. In a broad sense the strategies employed in the two works are similar, but they differ considerably in detail. Meyer et al. appeal to their variational principle in a much stronger way than we do to ours. We use the McLachlan principle only to help us pick a reasonable separable, global effective potential to guide all of our traveling basis functions. Indeed, in light of the observed practical equivalence of a number of variations on the (nonvariational) theme suggested in Eq. (4.1), it does not appear essential to use the McLachlan formalism for this purpose (although it is certainly comforting to have it available when one is approaching a novel problem). Once the basis set has been constructed, there is also no need to invoke the notion of variational optimization. We can regard the configuration interaction phase of our algorithm simply as an expansion of the exact wave packet in a complete basis set which happens to travel around the coordinate space.

Meyer et al. use the Lagrangian variational principle to couple all basis wave packets and all coefficients together in a complicated way. The evolution of each basis packet in each coordinate, e.g. $\varphi_{x}^{(j)}(x)$ for all $j$, is individually optimized, and, moreover, the optimization is done self-consistently with the evolution of optimized superposition coefficients. The advantage of this scheme is that the basis set is more accommodating than ours, since the basis functions in each coordinate are not restricted to be propagated under the same effective one dimensional interaction potential. There is also an explicit feedback mechanism not present in the method outlined in this work. Such a mechanism might correct errant Hartree-level wave packets before they have strayed too far from the course of the exact wave packet. The disadvantage of their scheme is that the equations of motion associated with it are much more complicated than ours: every basis function is linearly coupled to every other basis function in an intimate way. Thus, for a system with $D$ degrees of freedom, one has to evolve roughly $\Pi_{j=1}^{D} M_{j}$ coupled one-dimensional wave packets (where $\boldsymbol{M}_{j}$ is the number of 
basis functions used to span the $j$ th coordinate) in tandem with each other and with an equal number of basis coefficients. Consequently, their method does not appear to scale with the spatial dimensionality of the molecular system in as favorable way as ours.

In concluding we stress that from a global perspective the important point is that it is possible to add configuration interaction to Hartree-level wave packet dynamics. The algorithm presented in this work provides one promising way for obtaining precise information about the dynamics of many-body quantum systems, and judicious appeal to timedependent variational principles should enable refinement and specialization of the basic ideas case by case.

\section{ACKNOWLEDGMENTS}

J.C-M. gratefully acknowledges a grant from the Ministerio de Educacion y Ciencia of Spain and the Fullbright Commission. R.D.C. wishes to thank the National Science Foundation, the Donors of the Petroleum Research Fund, the Alfred P. Sloan Foundation and the Camille and Henry Dreyfus Foundation for financial support, and the Pittsburgh Supercomputer Center for supercomputer time.
'For details of bound state calculations relevant to molecular spectroscopy, see G. Herzberg, Molecular Spectra and Molecular Structure (Van Nostrand Reinhold, NY, 1939, 1945, 1966), Vols. 1, $2,3$.

${ }^{2}$ For representative coupled channel methods for inelastic molecular scattering problems, see W. L. Lester, J. Comput. Phys. 3, 22 (1968); Methods Comput. Phys. 10, 211 (1971).

${ }^{3}$ (a) E. J. Heller, J. Chem. Phys. 62, 1544 (1975); (b) Acc. Chem. Res. 14, 368 (1981); (c) S.-Y. Lee and E. J. Heller, J. Chem. Phys. 76, 3035 (1982); (d) K. C. Kulander and E. J. Heller, J. Chem. Phys. 69, 2439 (1978); (e) E. J. Heller, R. L. Sundberg, and D. J. Tannor, J. Phys. Chem. 86, 1822 (1982).

${ }^{4}$ See, for example, (a) J. Brickmann and P. Russeger, J. Chem. Phys. 75, 5744 (1981); (b) R. T. Skodje and D. G. Truhlar, J. Chem. Phys. 80, 3123 (1984); R. T. Skodje, Chem. Phys. Lett. 109, 221 (1984).

${ }^{5}$ R. D. Coalson and M. Karplus, Chem. Phys. Lett. 90, 301 (1982).

${ }^{6}$ R. D. Coalson, Chem. Phys. Lett. 165, 443 (1990).

${ }^{7}$ A. D. McLachlan, Mol. Phys. 8, 39 (1964).

${ }^{8}$ H.-D. Meyer, U. Manthe, and L. S. Cederbaum, Chem. Phys. Lett. 165, 73 (1990).

${ }^{9}$ See R. Kosloff, J. Phys. Chem. 92, 2087 (1988), for examples.

${ }^{10}$ M. Messina and R. D. Coalson, J. Chem. Phys. 90, 4015 (1989).

"D. Secrest and B. R. Johnson, J. Chem. Phys. 4, 4556 (1966).

${ }^{12}$ (a) M. D. Feit, J. A. Fleck, Jr., and A. Steiger, J. Comput. Phys. 47, 412 (1982); (b) N. Halbersadt, J. A. Beswick, and J. M. Launay (private communication).

${ }^{13}$ (a) J. Kucar, H.-D. Meyer, and L. S. Cederbaum, Chem. Phys. Lett. 140, 525 (1987); (b) H.-D. Meyer, J. Kucar, and L. S. Cedarbaum, J. Math. Phys. 29, 1417 (1988).

${ }^{14}$ Z. Kotler, A. Nitzan, and R. Kosloff, Chem. Phys. Lett. 153, 483 (1988); see also N. Makri and W. H. Miller, J. Chem. Phys. 87, 5781 (1987),

${ }^{15}$ B. Jackson and H. Metiu, J. Chem. Phys. 84, 3535 (1986).

${ }^{16}$ See any book on linear algebra, e.g., T. M. Apostol, Calculus, Vol. 2, 2nd edition (Xerox College Publishing, Waltham, MA, 1969).

${ }^{17}$ See Refs. 13 for a full discussion of this principle. 